\title{
छs \\ Cyclotrons with fast variable and/or multiple energy extraction
}

\author{
C. Baumgarten* \\ Paul Scherrer Institute, 5232 Villigen PSI, Switzerland
}

(Received 29 July 2013; published 24 October 2013)

\begin{abstract}
We discuss the possibility in principle of stripping extraction in combination with reverse bends in isochronous separate-sector cyclotrons (and/or fixed field alternating gradient accelerators). If one uses reverse bends between the sectors (instead of or in combination with drifts) and places stripper foils at the sector exit edges, the stripped beam has a reduced bending radius and it should be able to leave the cyclotron within the range of the valley-even if the beam is stripped at less than full energy. We are especially interested in stripping of $\mathrm{H}_{2}^{+}$, as it doubles the charge to mass ratio of the ions. However the method could be applied to other ions or ionized molecules as well. For the production of proton beams by stripping extraction of an $\mathrm{H}_{2}^{+}$beam, we discuss possible designs for three types of machines: First, a lowenergy cyclotron for the simultaneous production of several beams at multiple energies-for instance 15 , 30 , and $70 \mathrm{MeV}$ - thus allowing beam delivery on several isotope production targets. In this case it can be an advantage to have a strong energy dependence of the direction of the extracted beam. Second, we consider a fast variable-energy proton machine for cancer therapy that should allow extraction (of the complete beam) at all energies in the range of about $70 \mathrm{MeV}$ to about $250 \mathrm{MeV}$ into the same beam line. Third, we consider a high-intensity high-energy machine, where the main design goals are extraction with low losses, low activation of components, and high reliability. Especially if such a machine is considered for an accelerator driven system (ADS), this extraction mechanism has advantages: Beam trips by the failure of electrostatic elements could be avoided and the turn separation would be less critical, which allows operation at lower main cavity voltages. This would in turn reduce the number of $\mathrm{rf}$ trips. The price that has to be paid for these advantages is an increase in size and/or field strength compared to proton machines with standard extraction at the final energy.
\end{abstract}

DOI: 10.1103/PhysRevSTAB.16.100101

PACS numbers: 29.20.dg, 87.56.bd, 28.65.+a

\section{INTRODUCTION}

A major fraction of the practical problems in the operation of cyclotrons is related to beam extraction: the activation of extraction elements increases the personal dose during maintenance work and sometimes requires the beam to be shut off long before the scheduled work. The electrostatic elements are frequently the cause of beam interruptions due to high voltage trips; they require regular maintenance like cleaning and conditioning. In order to increase the extraction efficiency, the energy gain per turn must be maximized, which requires cavities and resonators to run at the limit of what can be achieved. This in turn increases the frequency of cavity trips and amplifier failures.

In this work, we propose to utilize the mechanism of stripping extraction first demonstrated with $\mathrm{H}^{-}$ions at the Colorado cyclotron [1] and fairly well established for various positive and negative ions in many machines worldwide in nearly the complete energy and intensity range that can be achieved by cyclotrons [2-8]. The extraction mechanism

\footnotetext{
*christian.baumgarten@psi.ch
}

Published by the American Physical Society under the terms of the Creative Commons Attribution 3.0 License. Further distribution of this work must maintain attribution to the author(s) and the published article's title, journal citation, and DOI. that we present here might help to avoid most extraction problems completely. In the case of variable energy extraction as we propose for proton therapy machines, energy degraders and energy selection systems can be omitted, thus reducing the costs and the facility footprint significantly. Since the required beam intensities are typically only in the order of $1 \mathrm{nA}$ at the patient, it should be possible to keep a cyclotron with variable energy extraction almost free from activation of components. Higher beam currents-of up to $1 \mu \mathrm{A}$ - are mainly required to compensate the losses of energy degradation and collimation [9].

Extraction of $\mathrm{H}^{-}$ions with variable energies was employed extensively first at the UCLA cyclotron [10], but also at the Manitoba cyclotron [11]. It is commonly used in industrial low-energy isotope production cyclotrons. Unfortunately, the $\mathrm{H}^{-}$ion is not stable in strong magnetic fields at high energy, so that $\mathrm{H}^{-}$cyclotrons are either limited in energy or restricted in magnetic field strength. This requires large radius machines like the TRIUMF cyclotron [12]. Furthermore, the use of $\mathrm{H}^{-}$ions in accelerators is more demanding with respect to the machine vacuum and the production of $\mathrm{H}^{-}$in ion sources.

Cyclotrons [and/or fixed field alternating gradient accelerators (FFAGs)] with reverse bends have been proposed in the past [13-15]—mainly in order to achieve the focusing conditions that are required for energies of $1 \mathrm{GeV}$ and 
above. However, there is no publication known to the authors that proposes the use of reverse bends in combination with stripping extraction.

In most (if not all) cases where stripping extraction of $\mathrm{H}_{2}^{+}$ions is used, the beam bends inwards and the proposed extraction schemes lead to complicated orbits that circle one or even multiple times within the cyclotron before the beam exits [2-8]. The use of this method for multiple or even for continuously variable energy extraction is difficult-if at all possible.

Another method to achieve beam extraction at variable energy is to vary the main field of the cyclotron and to use a sequence of trim coils to achieve isochronism for the desired extraction energy. This method is the most "natural" way and it is known to work [16]. However, the minimal time to switch between energies is given by the ramping of the main field and the magnetic relaxation time of the yoke. In the optimal case it might be possible to realize energy switching within minutes.

The RACCAM project [17] studies the possibility of fast energy variation from 70 to $180 \mathrm{MeV}$ by the use of a FFAG with laminated magnets. This implies the use of variable injection energy by a small injector cyclotron and variable energy $\mathrm{H}^{-}$-stripping extraction. The most obvious disadvantage of a RACCAM-like layout is the complexity and size of this system consisting of two accelerators with relatively complex injection and extraction mechanisms. It is unlikely that the RACCAM system is competitive to a separate-sector cyclotron as we propose below with respect to price, beam quality, and simplicity. Furthermore, the proposed RACCAM system has a pulsed beam (instead of $\mathrm{CW}$ ), a smaller energy range, and at least the same footprint.

The goal of this work is to present first basic geometrical and beam dynamical studies in order to investigate the feasibility of variable energy cyclotrons and to explore the energy ranges that could be achieved. Concerning the beam dynamics we restrict ourselves to the minimum, which we consider to be the verification of the stability of motion of a coasting beam and of the extraction mechanism. In order to survey the parameter space for such machines, we restrict ourselves to the so-called hard-edge approximation of the magnets. We further simplify this approach by assuming homogeneous magnetic fields within sectors and valleys. Isochronism is achieved exclusively by a variation of the azimuthal sector width along the orbit [18].

In Sec. II, we give a description of the geometry and the calculation of the transfer matrices. The equations given there have been used in MATHEMATICA ${ }^{\circledR}$ to analyze the orbits and the traces of the transfer matrices in hard-edge approximation in order to find stable solutions with the desired extraction orbits. Based on the results, a "C" program was used to generate smoothed magnetic field maps, which have then been analyzed with an equilibrium orbit code [19] and a Cartesian tracking code to numerically verify the analytical results of the hard-edge approximation.
In Secs. III, IV, and V we present the results of the calculations.

\section{GEOMETRY OF A SEPARATE-SECTOR CYCLOTRON WITH REVERSE BENDS}

We consider $\mathrm{H}_{2}^{+}$cyclotrons that are composed of $\mathrm{N}$ identical cells which are each composed of a sequence of homogeneous sector magnets, reverse bends with homogeneous fields, and (optionally) drifts. We do not consider beam injection nor other details of central regions. We are not concerned about the question, whether these machines might need preaccelerators or can be made "compact." We first consider machines that are composed of exclusively positive and negative bends as shown in Fig. 1, where we call the positive bends "sector" and the negative bends "valley."

The absolute values of the sector (valley) field is $B_{s}\left(B_{v}\right)$, the corresponding bending angle is $2 \phi_{s}\left(2 \phi_{v}\right)$, then we have for an ion of mass $m$, charge $q$ and, momentum $p=m c \gamma \beta$ :

$$
\begin{aligned}
\frac{\pi}{N} & =\phi_{s}-\phi_{v}, \quad R_{s}=\frac{p}{q B_{s}}=\frac{m c \gamma \beta}{q B_{s}}, \quad R_{v}=\frac{p}{q B_{v}}=\frac{m c \gamma \beta}{q B_{v}}, \\
L_{\mathrm{tot}} & =2 N\left(R_{s} \phi_{s}+R_{v} \phi_{v}\right)=2 N \frac{m c}{q}\left(\frac{\phi_{s}}{B_{s}}+\frac{\phi_{v}}{B_{v}}\right) \beta \gamma .
\end{aligned}
$$

Isochronism requires that the velocity $v$, the orbital angular frequency $\omega_{o}=\frac{2 \pi}{T}$, and the total length of the orbit $L_{t o t}$ are related by

$$
v=\frac{L_{\mathrm{tot}}}{T}=\frac{\omega_{o} L_{\mathrm{tot}}}{2 \pi}, \quad \beta=\frac{v}{c}=\frac{\omega_{o} L_{\mathrm{tot}}}{2 \pi c}=\frac{L_{\mathrm{tot}}}{2 \pi a},
$$

where $a=\frac{c}{\omega_{o}}$ is the cyclotron length unit. In combination with Eq. (1) this yields

$$
\begin{aligned}
2 \pi a \beta & =2 N \frac{m c}{q}\left(\frac{\phi_{s}}{B_{s}}+\frac{\phi_{v}}{B_{v}}\right) \beta \gamma, \\
a & =\frac{N}{\pi} \frac{m c}{q}\left(\frac{\phi_{s}}{B_{s}}+\frac{\phi_{s}-\pi / N}{B_{v}}\right) \gamma, \\
\phi_{s} & =\frac{\pi}{N(1+\lambda)}\left(\frac{B_{v}}{B_{0} \gamma}+1\right),
\end{aligned}
$$

where we used $\lambda=\frac{B_{v}}{B_{s}}=\frac{R_{s}}{R_{v}}$ and defined the "nominal field" $B_{0}$ by

$$
B_{0}=\frac{m c}{a q}=\frac{m}{q} \omega_{o}
$$

From Fig. 1, we pick the following equations:

$$
\begin{aligned}
R_{v} \sin \left(\phi_{v}\right) & =R \sin \left(\frac{\pi}{N}-\frac{\alpha}{2}\right) \\
& =R\left[\sin \left(\frac{\pi}{N}\right) \cos \left(\frac{\alpha}{2}\right)-\cos \left(\frac{\pi}{N}\right) \sin \left(\frac{\alpha}{2}\right)\right], \\
R_{s} \sin \left(\phi_{s}\right) & =R \sin \left(\frac{\alpha}{2}\right),
\end{aligned}
$$




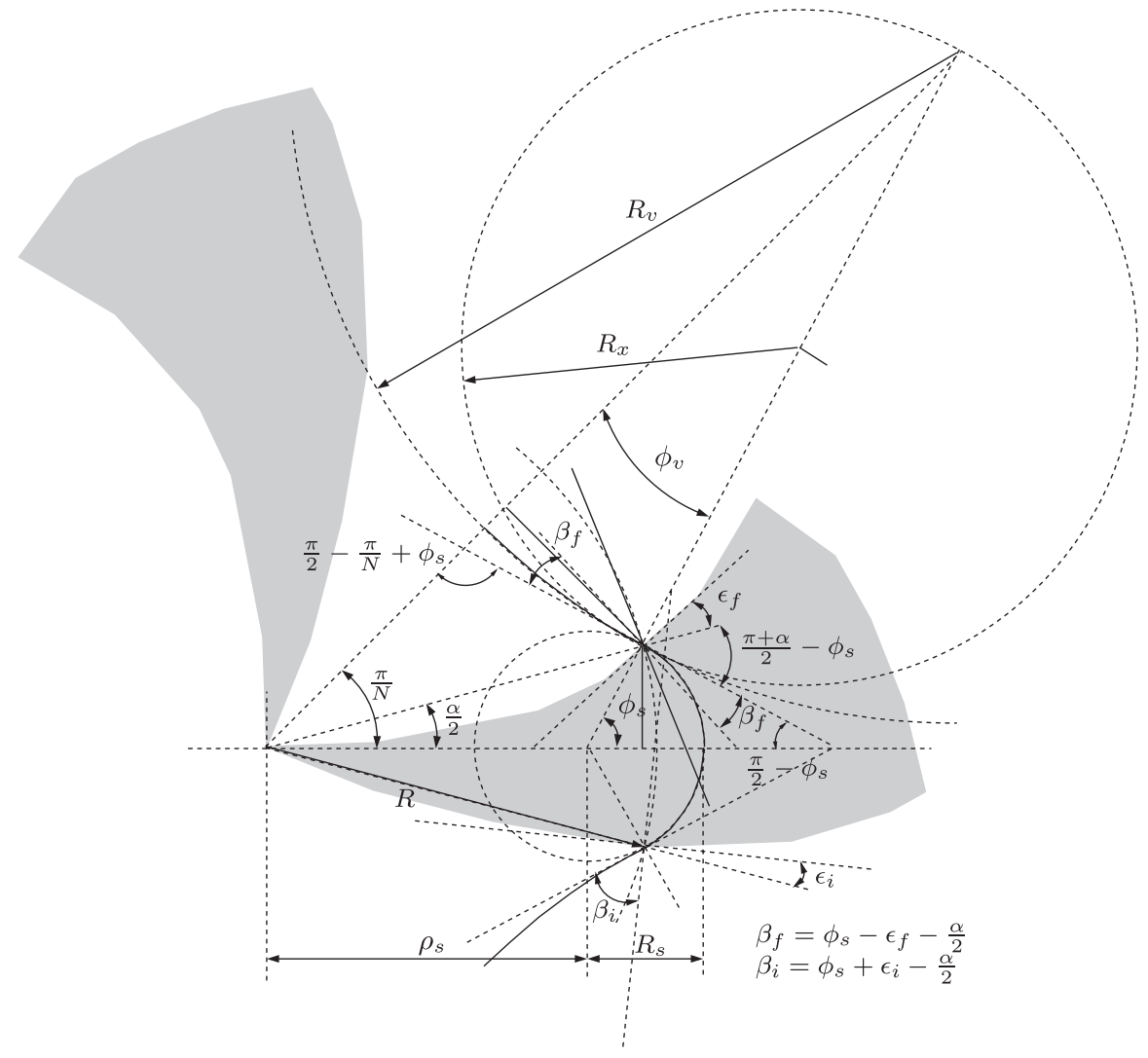

FIG. 1. Geometry of a cyclotron cell with reverse bends. The spiralled sector is indicated by a gray polygon. Since it has a constant field, the equilibrium orbit for a certain energy is composed of two arcs: Within the sector magnet it has bending radius $R_{s}$ and within the reverse bend (valley), it has a larger radius $R_{v}$.

where $R$ is the radius of the orbit entering (exiting) the sector. From this we obtain in a few steps

$$
\tan \left(\frac{\alpha}{2}\right)=\frac{\lambda \tan \left(\frac{\pi}{N}\right) \tan \left(\phi_{s}\right)}{(1+\lambda) \tan \left(\phi_{s}\right)-\tan \left(\frac{\pi}{N}\right)} .
$$

If $\theta_{c}$ is the azimuthal angle of the sector center and $\theta_{i}\left(\theta_{f}\right)$ are the angles of entrance (exit) of the orbit into the sector, then

$$
\theta_{i}=\theta_{c}-\frac{\alpha}{2}, \quad \theta_{f}=\theta_{c}+\frac{\alpha}{2} .
$$

The angles $\varepsilon_{i}\left(\varepsilon_{f}\right)$ between the sector edges and the radial direction can be obtained by

$$
\begin{aligned}
& \tan \varepsilon_{i}=R \frac{d \theta_{i}}{d R}=R \frac{d \theta_{i}}{d \gamma} / \frac{d R}{d \gamma}, \\
& \tan \varepsilon_{f}=R \frac{d \theta_{f}}{d R}=R \frac{d \theta_{i}}{d \gamma} / \frac{d R}{d \gamma} .
\end{aligned}
$$

The angles between the orbit and the sector edges (which are required for the transfer matrices) can be computed by

$$
\beta_{i}=\phi_{s}+\varepsilon_{i}-\frac{\alpha}{2}, \quad \beta_{f}=\phi_{s}-\varepsilon_{f}-\frac{\alpha}{2} .
$$

The radii of the arc centers in the sector $\rho_{s}$ and the valley $\rho_{v}$ are
$\rho_{s}=R_{s}\left(\frac{\sin \phi_{s}}{\tan \left(\frac{\alpha}{2}\right)}-\cos \phi_{s}\right), \quad \rho_{v}=R_{v}\left(\frac{\sin \phi_{v}}{\tan \left(\frac{\pi}{N}-\frac{\alpha}{2}\right)}+\cos \phi_{v}\right)$.

The Cartesian coordinates $x_{s}(\phi), y_{s}(\phi)$ of the orbit inside the sector in dependence of the angle $\phi$ can be written as

$$
\begin{aligned}
& x_{s}(\phi)=\left(\rho_{s}+R_{s} \cos \phi\right) \cos \theta_{c}-R_{s} \sin \phi \sin \theta_{c}, \\
& y_{s}(\phi)=\left(\rho_{s}+R_{s} \cos \phi\right) \sin \theta_{c}+R_{s} \sin \phi \cos \theta_{c},
\end{aligned}
$$

where $\phi$ ranges from $-\phi_{s}$ to $\phi_{s}$. Correspondingly, one finds for the valley

$$
\begin{aligned}
& x_{v}(\phi)=\left(\rho_{v}-R_{v} \cos \phi\right) \cos \theta_{c}-R_{v} \sin \phi \sin \theta_{c} \\
& y_{v}(\phi)=\left(\rho_{v}-R_{v} \cos \phi\right) \sin \theta_{c}+R_{v} \sin \phi \cos \theta_{c}
\end{aligned}
$$

where $\phi$ ranges from $-\phi_{v}$ to $\phi_{v}$.

If we assume that a stripper foil is placed exactly at the sector exit (i.e. at radius $R$ and azimuthal angle $\theta_{f}$ ), then the center coordinates $x_{c}(\phi), y_{c}(\phi)$ of the arc described by the extracted orbit are 


$$
\begin{aligned}
x_{c}= & \left(\rho_{s}+\left(R_{s}+R_{v} / 2\right) \cos \phi_{s}\right) \cos \theta_{c} \\
& -\left(R_{s}+R_{v} / 2\right) \sin \phi_{s} \sin \theta_{c}, \\
y_{c}= & \left(\rho_{s}+\left(R_{s}+R_{v} / 2\right) \cos \phi_{s}\right) \sin \theta_{c} \\
& +\left(R_{s}+R_{v} / 2\right) \sin \phi_{s} \cos \theta_{c}
\end{aligned}
$$

coordinates $x_{x}(\phi), y_{x}(\phi)$ of the extracted orbit are

$x_{x}(\phi)=x_{c}+R_{v} / 2 \cos \left(\theta_{c}+\phi_{s}+\pi-\phi\right)$,

$y_{x}(\phi)=y_{c}+R_{v} / 2 \sin \left(\theta_{c}+\phi_{s}+\pi-\phi\right)$,

where $\phi$ starts at zero. With the above equations, we analyzed the geometry of the orbit and the extraction for different choices of $B_{s}, B_{v}, B_{0}$, and $\theta_{c}(\gamma)$ as a function of $\gamma=1+E / E_{0}$.

Because of the increasing complexity of a graphical analysis of the geometry we present an ansatz for an algorithmic method in the Appendix. In a simple situation such as a separated-sector cyclotron the drawing might do as well. But in the case of the medical cyclotron discussed below, the symmetry of the equilibrium orbit for a given energy is broken by the drift between the reverse bend (valley) and the next sector. In this case and those of more complex configurations, the analysis of the layout by a hand-made sketch becomes cumbersome due to the increasing number of angles and geometrical relations. In fact, the geometry of the medical cyclotron presented below has been analyzed by a " $\mathrm{C}$ " program and a MATHEMATICA ${ }^{\circledR}$ notebook based on the algebraic ansatz of the Appendix. The main reason was the desire to create a map of the magnetic field in cylindrical coordinates for the numerical (and hence more "realistic") computation of the tunes. In the case of a cyclotron that is composed of $N$ cells each containing a sector magnet, a reverse bend, and a drift, it turns out that the entrance and exit radius for a given energy are not equal.

For a given radius of the polar magnetic field grid, we had to determine the energy (i.e. the $\gamma$ value) of the equilibrium orbit entering the sector, a second $\gamma$ value for the orbit exiting the sector, and a third one at the exit of the reverse bend. This was done by an iterative numerical interval search.

\section{The transfer matrices}

The horizontal transfer matrices $\mathbf{M}_{s, v}$ for the sector (valley) are given by

$$
\mathbf{M}_{s, v}=\left(\begin{array}{cc}
\cos \left(2 \phi_{s, v}\right) & R_{s, v} \sin \left(2 \phi_{s, v}\right) \\
-\frac{\sin \left(2 \phi_{s, v}\right)}{R_{s, v}} & \cos \left(2 \phi_{s, v}\right)
\end{array}\right) .
$$

The horizontal transfer matrix that describes the edge focusing effect is

$$
\mathbf{M}_{i, f}=\left(\begin{array}{cc}
1 & 0 \\
\frac{\tan \left(\beta_{i, f}\right)}{R_{\mathrm{eff}}} & 1
\end{array}\right),
$$

where $R_{\text {eff }}=\left(R_{s}^{-1}+R_{v}^{-1}\right)^{-1}$. Starting with the entrance into the sector magnet, the horizontal transfer matrix $\mathbf{M}$ for a single section is the product

$$
\mathbf{M}_{\mathrm{sec}}=\mathbf{M}_{v} \mathbf{M}_{f} \mathbf{M}_{s} \mathbf{M}_{i} .
$$

The radial focusing frequency $\nu_{r}$ can be obtained from a parametrization by the parameters $\alpha_{t}, \beta_{t}$, and $\gamma_{t}$ :

$$
\mathbf{M}_{\mathrm{sec}}=\mathbf{1} \cos \left(2 \pi \nu_{r}\right)+\left(\begin{array}{cc}
\alpha_{t} & \beta_{t} \\
-\gamma_{t} & -\alpha_{t}
\end{array}\right) \sin \left(2 \pi \nu_{r}\right),
$$

from which one obtains with $\beta_{t} \gamma_{t}-\alpha_{t}^{2}=1$

$$
\begin{aligned}
\cos \left(2 \pi \nu_{r}\right) & =\operatorname{Tr}\left(\mathbf{M}_{\mathrm{sec}}\right) / 2 \sin \left(2 \pi \nu_{r}\right)^{2} \\
& =\operatorname{Det}\left[\mathbf{M}_{\mathrm{sec}}-\mathbf{1} \operatorname{Tr}\left(\mathbf{M}_{\mathrm{sec}}\right) / 2\right] .
\end{aligned}
$$

The matrices for the vertical motion are

$$
\mathbf{T}_{s, v}=\left(\begin{array}{cc}
1 & 2 R_{s, v} \phi_{s, v} \\
0 & 1
\end{array}\right), \quad \mathbf{T}_{i, f}=\left(\begin{array}{cc}
1 & 0 \\
-\frac{\tan \left(\beta_{i, f}\right)}{R_{\mathrm{eff}}} & 1
\end{array}\right),
$$

so that correspondingly

$$
\mathbf{T}_{\mathrm{sec}}=\mathbf{T}_{v} \mathbf{T}_{f} \mathbf{T}_{s} \mathbf{T}_{i}
$$

The motion is stable if

$$
\left|\operatorname{Tr}\left(M_{\text {sec }}\right) / 2\right| \leq 1, \quad\left|\operatorname{Tr}\left(T_{\text {sec }}\right) / 2\right| \leq 1 .
$$

In the case of cyclotrons with reverse bends, one has a huge flutter $F=\frac{\left\langle B^{2}\right\rangle-\langle B\rangle^{2}}{\langle B\rangle^{2}}$ due to the negative field regions. If one uses the dimensionless ratios $\lambda=\frac{B_{v}}{B_{s}}=\frac{R_{s}}{R_{v}}$ and $\mu=\frac{B_{0}}{B_{s}}$, then the flutter yields

$$
F=\frac{(1-\mu \gamma)(\mu \gamma+\lambda)}{\mu^{2} \gamma^{2}},
$$

and can easily approach values of above 4 . Therefore care must be taken to not have too strong focusing, i.e., to avoid the $N / 2$ stop band. Because of the huge flutter, the spiral angle must be kept sufficiently small to avoid the stop band.

\section{A MULTIBEAM ISOTOPE PRODUCTION CYCLOTRON}

As already mentioned, many isotope production machines with variable energy extraction by stripping of $\mathrm{H}^{-}$ ions are in use. Because of the lower charge to mass ratio $\mathrm{H}_{2}^{+}$machines are larger by a factor of 2 (or more) compared to $\mathrm{H}^{-}$cyclotrons at the same field strength. Nevertheless at an energy of $70 \mathrm{MeV}$ the beam loss per meter by Lorentz stripping for fields of $2.1 \mathrm{~T}$ reaches the percent level [20]. Also residual gas stripping has to be considered at higher energies due to the nonlinear increase of the path length with energy. The pressure must be less than $5 \times 10^{-8}$ Torr in order to achieve losses below $2 \%$ in the CYCIAE-100 [20]. Therefore the use of $\mathrm{H}_{2}^{+}$-stripping extraction with reverse bends might be of interest for high 


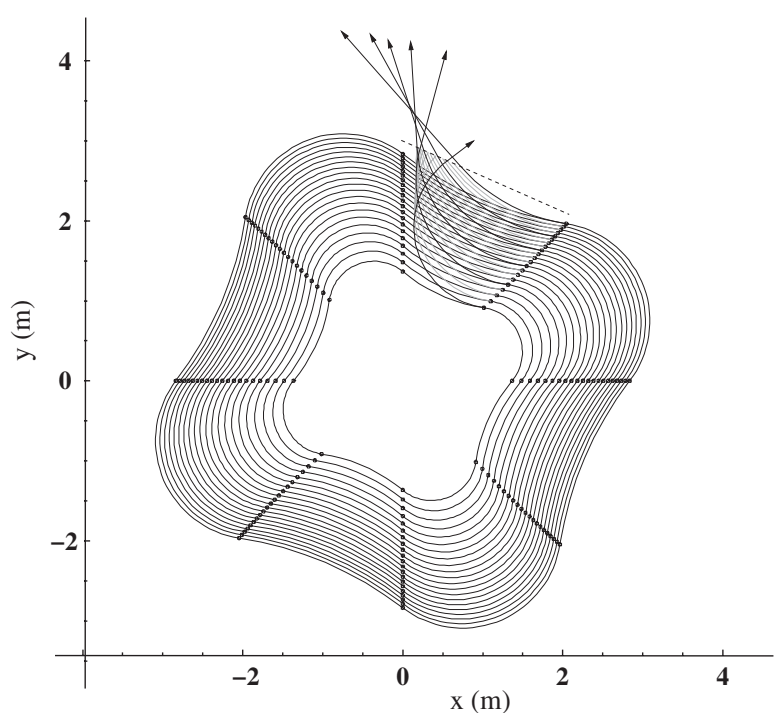

FIG. 2. Geometry of a $\mathrm{H}_{2}^{+}$cyclotron for isotope production with reverse bends. The $\mathrm{H}_{2}^{+}$beam is stripped at the sector edge (indicated by symbols). The orbits of stripped proton beams are shown from 15 to $70 \mathrm{MeV}$ in steps of $2.75 \mathrm{MeV}$. The arrows indicate the extracted beams for 15, 26, 37, 48, 59, and $70 \mathrm{MeV}$. The dashed line is the field edge of the reverse bend.

current low loss isotope production machines at energies well above $30 \mathrm{MeV}$.

The simplified cyclotron model above allows an initial diagnosis whether extraction at various energies can be combined with stability of axial and horizontal motion. Figure 2 shows a plan view of an isotope production machine with maximal $\mathrm{H}_{2}^{+}$energy of $140 \mathrm{MeV}$ that allows stripping extraction of proton beams with energies between 15 and $70 \mathrm{MeV}$. The nominal field $B_{0}$ is $0.75 \mathrm{~T}$, the sector field $B_{s} 2 \mathrm{~T}$, and the field strength $B_{v}$ in the reverse bends is $0.55 \mathrm{~T}$. Without further provision, the directions of the extracted beams differ enough to allow for energy-specific targets. If the beam is partially stripped at lower energy, simultaneous irradiation at several targets should be possible. If superconducting coils are used to increase the field strength, the size of the cyclotron decreases accordingly. The sector entrance edge has been chosen straight $\frac{d \theta_{i}}{d \gamma}=0$ so that $\theta_{c}=\theta_{i}+\alpha / 2$ and $\theta_{f}=\theta_{i}+\alpha$.

Figure 3 shows the corresponding tune diagram. The radial tune is (except for the last turns) approximately constant at about $\nu_{r} \approx 1.5$. The vertical tune $\nu_{z}$ starts at low energy at about 1.9 and decreases smoothly to about 1.75 at maximal energy. The tails of the tune are an artifact from the magnetic field interpolation close to the maximum radius. Because of the strong flutter, the axial tune $\nu_{z}$ is very large. As a consequence the minimum number of sectors is likely 4 , so that the $N / 2$ stop band starts at $\nu=2$. Higher sector numbers are possible in principle, but more expensive and not required for this energy range. Partial stripping of the beam could allow simultaneous extraction at multiple energies. For this purpose one would move a

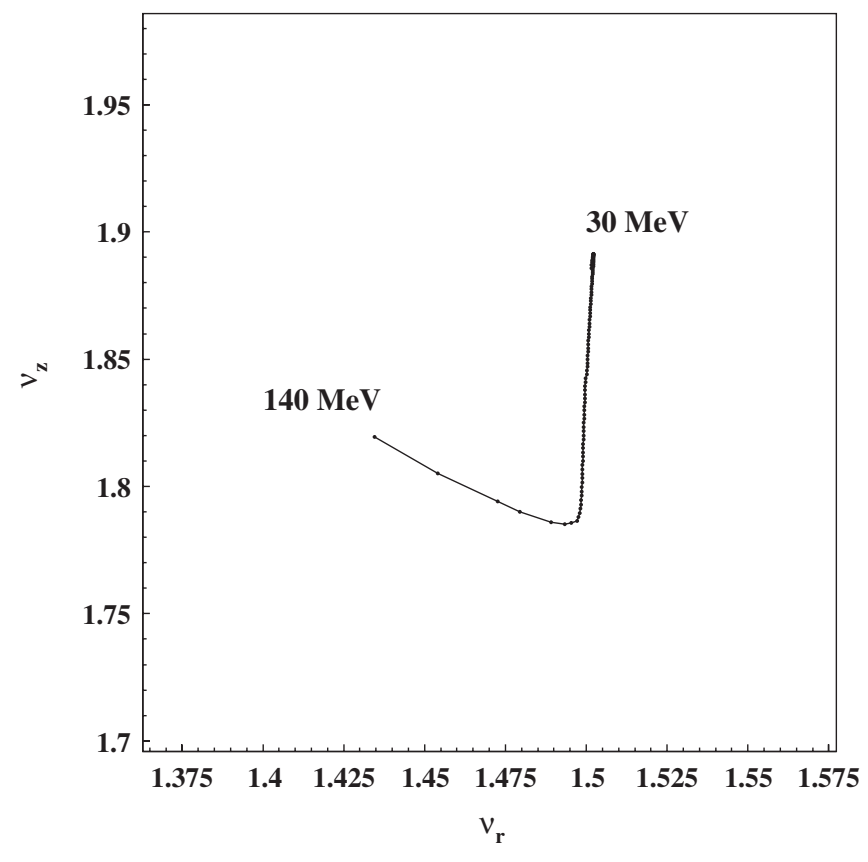

FIG. 3. Tunes of the isotope production cyclotron for $\mathrm{H}_{2}^{+}$ energies from 30 to $140 \mathrm{MeV}$.

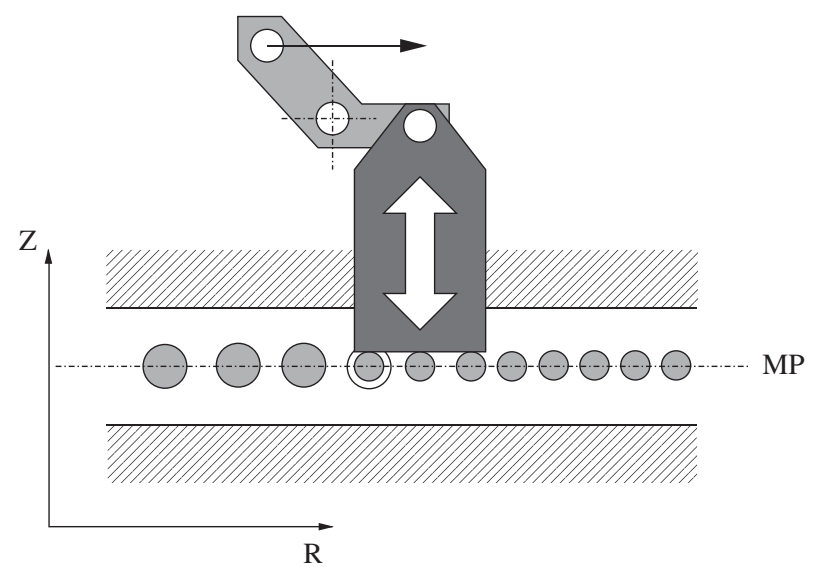

FIG. 4. Partial beam stripping by vertical positioning of a stripper foil keeping a certain distance to the median plane (MP).

stripper foil vertically towards the median plane until it strips off the desired beam current for the corresponding energy (see Fig. 4). The remaining beam (with reduced emittance) may be accelerated to higher energies.

\section{A VARIABLE-ENERGY CYCLOTRON FOR PROTON THERAPY}

Commercially available cyclotrons for proton therapy typically deliver beams with an energy of 235-250 MeV $[21,22]$. Since the presently available cyclotron technology delivers the beam at fixed energy, the energy must be reduced to the value that is required for the treatment. This is typically done by energy degradation at the cost 
of significant emittance increase and energy straggling in the degradation process [23-25]. In order to fit to the acceptance of the beam transport system and to deliver a beam of the required quality most of the degraded beam has to be cut off by collimators and an energy selection system (ESS). The intensity is (depending on energy) reduced by up to 3 orders of magnitude.

Even though there are strong arguments for the use of cyclotrons in proton therapy, there are also disadvantages to the combination of a fixed-energy cyclotron, degrader and ESS: (1) the strong energy dependence of the beam intensity makes a fast and reliable energy variation (without intensity variation) of the beam difficult too achieve; (2) the activation of the accelerator, the degrader material, the collimators, and other components, which could be reduced by orders of magnitude, if one could extract high quality beam over the desired range of energies; (3) the cost for the degrader and the ESS, which typically consists of two dipoles, eight quadrupoles, movable slits, beam diagnostics, and vacuum components for about $10 \mathrm{~m}$ beam line; (4) the need to use large aperture quadrupoles and dipoles in order to achieve an adequate transmission efficiency for the beam line and gantry. The list is certainly incomplete, but it suffices to argue that one has to take the overall costs of an accelerator concept into account. A separate-sector cyclotron with reverse bends is certainly more expensive than a compact cyclotron. It will also have a larger footprint and a higher power consumption. However, the footprint of the accelerator itself is only a small fraction of a complete proton therapy facility.

We found that variable energy extraction by a movable stripper foil before a reverse bend allows for proton beam extraction in the range between 70 and $250 \mathrm{MeV}$. Figure 5 shows the layout of an $\mathrm{H}_{2}^{+}$cyclotron with $2 \mathrm{~T}$ sector magnets, a nominal field $B_{0}$ of 0.7 and $0.55 \mathrm{~T}$ reverse bend magnets, the equilibrium, and extraction orbits for energies from 70 to $250 \mathrm{MeV}$. With superconducting coils the field could be increased and size reduced by a factor of about 2 .

The tunes of the medical cyclotron are shown in Fig. 6 . They have been computed numerically together with the equilibrium orbits [19], since the symmetry of the geometry was broken due to the additional drifts of variable length. The drifts have been inserted in order to bring the extracted trajectories closer together as desired for variable energy extraction into a single beam line. With a bit more fine-shaping of the magnetic field of the reverse bend, it should be possible to make all extracted beams pass a region small enough to install a fast "catcher" magnet to steer the extracted beams of all energies into the same beam line. The small spiral angle has been introduced to avoid the $\nu_{r}=\nu_{z}$ resonance shown as a solid straight line. The oscillations at high energy are an artifact related to the interpolation close to the field edge. Using superconducting coils and correspondingly higher field values, the size

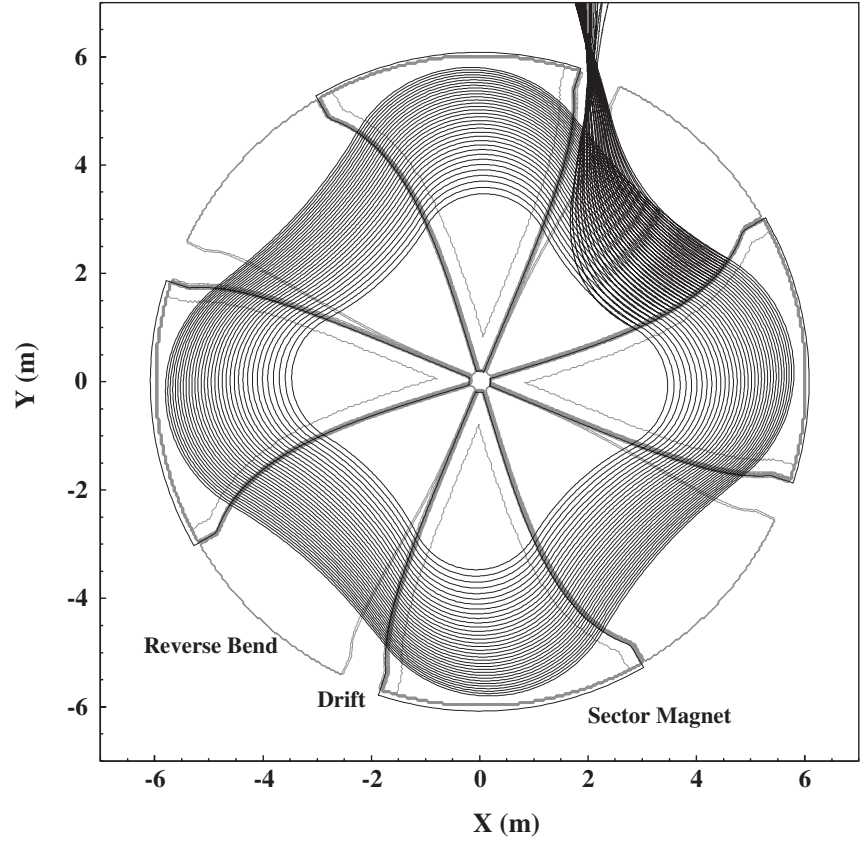

FIG. 5. Magnetic field contours of a $\mathrm{H}_{2}^{+}$cyclotron for proton therapy with reverse bends and drifts. The equilibrium orbits and stripped proton trajectories for energies from 70 to $250 \mathrm{MeV}$ in steps of $\approx 5.5 \mathrm{MeV}$ are plotted on top.

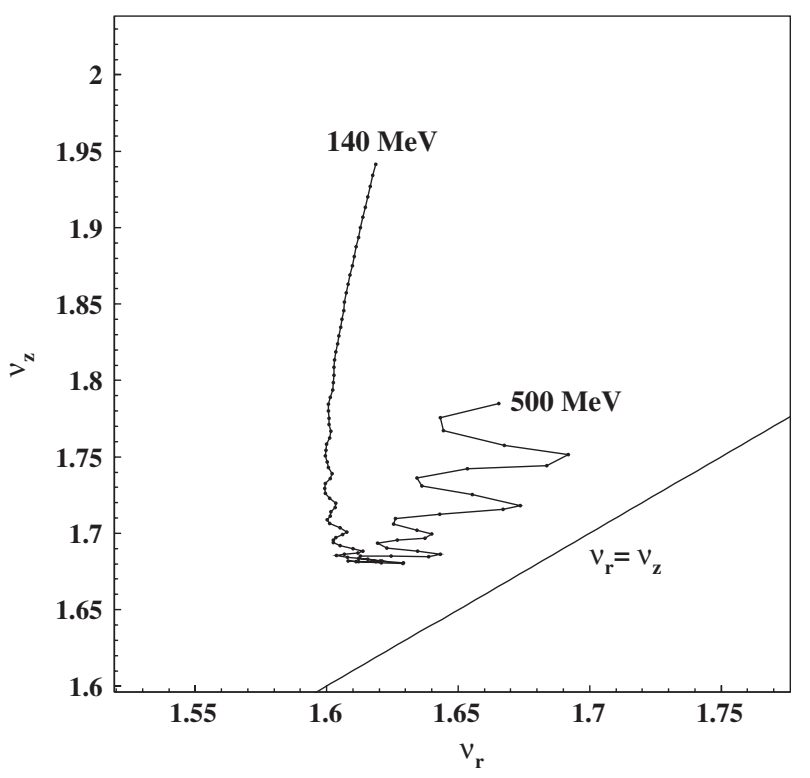

FIG. 6. Tune diagram of the medical cyclotron with variable energy extraction shown in Fig. 5.

could be reduced accordingly. If partial stripping would be applied, it should be possible to extract up to four beams simultaneously.

The time required for a change of the beam energy is then determined by the ramping time of the beam line magnets and the time for positioning the stripper foil. If a 
series of foils at different radii would be inserted vertically into the beam, then the actuator would need just a few millimeters of motion for the insertion of the foil as shown in Fig. 4. Other mechanisms using radial motion with the advantage of continuous energy adjustment are also possible. Even though the design of fast movable parts in vacuum is not trivial, we believe that mechanisms should be feasible with a response time in the order of $100 \mathrm{~ms}$ or below. Since the extracted beam current that is required for radiation therapy is of the order of $1 \mathrm{nA}$, cooling of the stripper foil is (for this application) not necessary.

More challenging (in terms of costs and engineering time) is the design of a central region with an internal ion source or a spiral inflector. An internal ion source causes a higher residual gas pressure compared to an external source. However, the beam current in such a proton therapy machine is very low so that even a high relative beam loss by residual gas stripping could be accepted.

Certainly the proposed extraction mechanism could also be used in combination with a preaccelerator, but the stripping process can be used only once. The preaccelerator would necessarily have a different extraction mechanism.

The layout shown in Fig. 5 has four sectors so that with an appropriate design of rf resonators one might use at maximum four exit ports in four directions. They might (but do not have to) be used simultaneously in order to deliver beam for four treatment rooms located around the cyclotron bunker. Since a direct beam from the cyclotron has a small emittance and energy spread, the beam transport system does not require magnets with large aperture. Hence, beam line and gantry might be smaller and cheaper than those of conventional systems. If the beam size and energy spread are too small for fast painting of the tumor, one could insert scatterers into the beam path-or one might directly use "thick" stripper foils, which increase the beam size by scattering and make the beam shape more Gaussian.

We used the flat field approach since it facilitates analytic calculations of the desired properties in very good approximation. However a cyclotron with a flat field has also practical advantages. Precision online field measurements by NMR probes might be possible if the field is sufficiently constant. Such measurements could be used to stabilize the magnetic field without beam extraction as is required for a phase probe [26]. This would not only reduce startup time and simplify beam quality management, but it might also reduce activation of an external beam dump. Furthermore the mechanism that places the stripper foil-if fast enough—could be made "fail safe": if a spring retracts the foil off the median plane in case of emergency, extraction immediately stops. Without the stripper foil and with an appropriate shaping of the edge field with enough phase shift per turn, the cyclotron could operate in a stand-by mode without activation and extraction but with continuous beam in the median plane. The beam would be accelerated to maximal energy, phase shifted in the fringe field, decelerated back to the cyclotron center, and dumped there without activation of components. In this way, the equipment could stay "warm" in stand-by mode. If beam is requested, the only action to be taken is to insert the stripper foil at the desired location for the requested energy.

\section{A HIGH-ENERGY HIGH-INTENSITY PROTON CYCLOTRON}

Recently there has been renewed interest in highintensity cyclotrons for accelerator driven systems (ADS) for transmutation of nuclear waste or as an "energy amplifier" [27] and also for physical experiments like $D A E \delta A L U S[4,28,29]$. Typically the cyclotron should be able to deliver $10 \mathrm{~mA}$ or more proton beam current at energies between 800 and $1000 \mathrm{MeV}$. Such cyclotrons have never been built, but the PSI ring machine which delivers $2.2 \mathrm{~mA}$ at $590 \mathrm{MeV}$ often serves as a proof-ofprinciple machine [30]. However, there is still a factor of 8 between the beam power of the PSI machine (1.3 MW) and the desired $10 \mathrm{MW}$ (or more) for an ADS driver. We are not going to discuss this in detail here, but we give an example of an $\mathrm{H}_{2}^{+}$cyclotron with stripping extraction between 500 and $950 \mathrm{MeV}$. The major advantage of stripping extraction is the increased reliability compared to extraction by electrostatic elements. Furthermore, the turn separation is not the limiting factor with respect to losses so that the voltage of the accelerating cavities is not a major design issue.

Figure 7 shows a machine layout for a homogenous sector (valley) field of $4 \mathrm{~T}(1.05 \mathrm{~T})$. The nominal field $B_{0}$

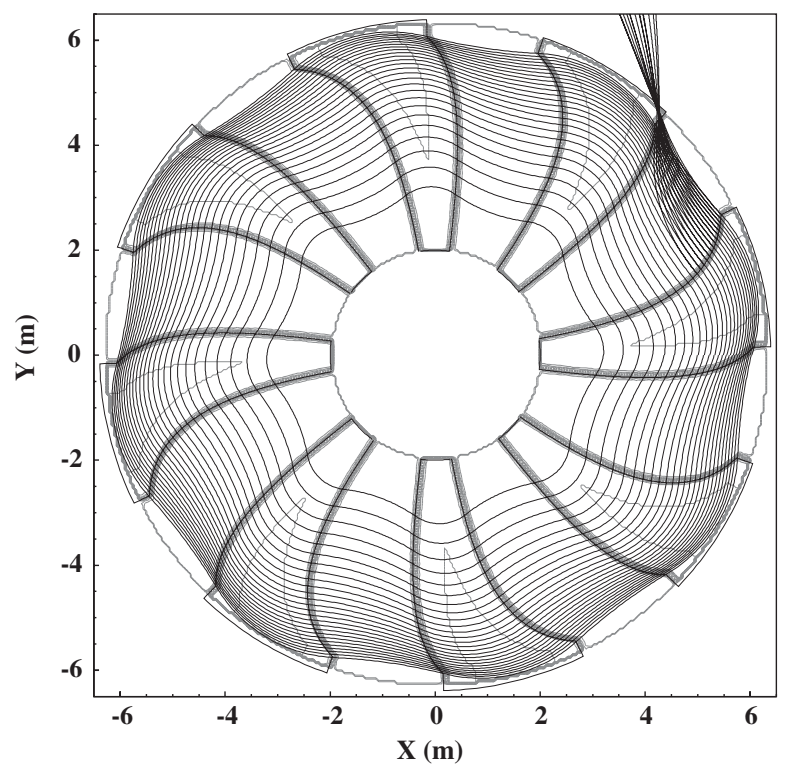

FIG. 7. Magnetic field contours, equilibrium orbits, and extraction trajectories of an eight-sector $\mathrm{H}_{2}^{+}$cyclotron for proton energies in the range between 500 and $950 \mathrm{MeV}$ for ADS. The orbits of the protons after stripping are shown from 470 to $950 \mathrm{MeV}$. 


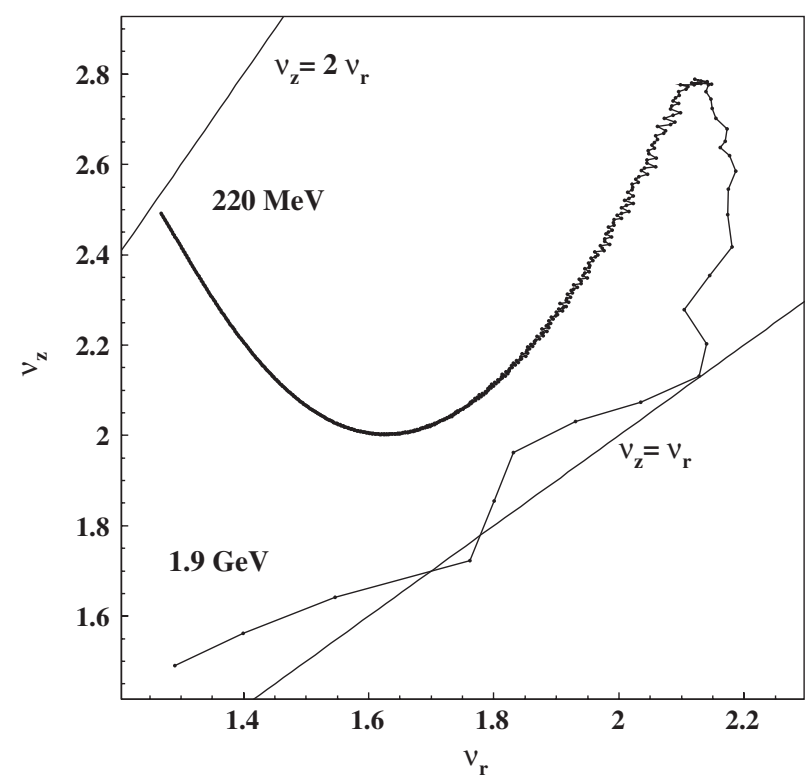

FIG. 8. Tune diagram of the high-intensity cyclotron with variable energy extraction shown in Fig. 7.

is $0.88 \mathrm{~T}$. The tune diagram is shown in Fig. 8 and covers the $\mathrm{H}_{2}^{+}$-energy range from $220 \mathrm{MeV}$ to $1.9 \mathrm{GeV}$. The spiral angle has been optimized to avoid major resonances. A more advanced field shaping with reduced flutter at lower energies would keep the vertical $\nu_{z}$ below $2 \nu_{r}$ at lower injection energy and would allow it to stay above $\nu_{r}$ at higher energies. The spiral angle has been chosen to be $\theta_{c}=(\gamma-1) / 4.2, \theta_{i}=\theta_{c}-\alpha / 2$, and $\theta_{f}=$ $\theta_{c}+\alpha / 2$. There are two major differences between the cyclotron design here and the one proposed in Ref. [4], the first being the difference in the vertical tune, which is in our design considerably increased by the reverse bends. The second is the trajectory of the stripped beam. The design proposed in Ref. [4] uses the conventional scheme in which the stripped beam is bent inwards and passes the cyclotron median plane at nearly all radii before exiting the field. There is no problem in principle with this scheme, but it has disadvantages. First the exact position and direction of the extracted beam depend on the cyclotron field all along the extraction orbit, which is more or less the complete median plane area. It is therefore influenced by trim coils, cavities, and main field changes. Second, this beam path has to be free from obstacles. The extraction path with reverse bends is as short as possible and passes only the area between two sectors. It is therefore less sensitive to changes of main field and/or trim coil settings.

If variable energy extraction is not required, then the reverse bends could likely be reduced to return flux gullies [14] plus drift space thus giving more space for rf cavities, pumping ports, or diagnostics. A reduced azimuthal width of the reversed bends would also allow the field strength (and/or) size of the sector magnets to be reduced.

\section{SOME FINAL REMARKS}

The machine layouts discussed allow some further optimization with respect to the direction of the extracted beam by an appropriate shaping of the fields of the sector magnets and the reverse bends. This would go beyond the scope of this paper, since our intention is to survey the possibilities of the extraction mechanism in principle. Certainly the flat field approach used above is neither necessary for this extraction scheme to work nor do we consider it to be the optimal choice. It has been chosen as it permits a fairly simple analytical description of the cyclotron beam optics.

The "inner region" of such cyclotrons, i.e., the energy range in which beam extraction is not possible, might be designed very differently from what is sketched above. The negative field in the reverse bends is not required at small radii. Therefore it is possible (and unavoidable) to reduce the effect of the reverse bend towards the cyclotron center (compensating this with reduced sector field or sector width).

The beam in a cyclotron like the ones described above should be centered so that the energy and radius are related in a predictable and reproducible way. This is especially important for proton therapy applications.

Since resonant beam extraction [31] is not required, the beam phase may be chosen flat for all energies. This allows for acceleration of beams with relatively low cavity voltages.

Since neither a low-energy spread nor a high turn separation is essential in order to minimize extraction losses (depending on the acceptance of the beam line transporting the beam to target), even the high-intensity machine might be operated without a flattop cavity. The space saved this way could be used to improve the vacuum conditions by the installation of cryogenic pumps. The beam loss by residual gas stripping has to be minimized when such cyclotrons are operated with high currents.

We have discussed a long list of advantages of the new extraction mechanism, but the method has its price: $\mathrm{H}_{2}^{+}$has half the charge to mass ratio of protons and therefore one has to double the size or field strength to reach the same final proton energy. The use of reverse bends has a similar effect. A discussion of if and when the increase in size or field strength pays off by the advantages is beyond the scope of this paper, but will depend on the purpose of the machine.

\section{SUMMARY}

The geometry of cyclotrons with reverse bends has been analyzed and the resulting transfer matrices have been given. We have investigated some of the design options involving the use of reverse bends in combination with stripping extraction of $\mathrm{H}_{2}^{+}$. We proved the feasibility in principle of variable energy/multiple beam extraction from cyclotrons with reverse bends and verified the analytical beam stability by a numerical calculation of the tunes.

We presented three potential applications for the extraction mechanism described: an isotope production cyclotron 
with simultaneous extraction at several energies between 15 and $70 \mathrm{MeV}$, a medical cyclotron with variable energy extraction in the range between 70 and $250 \mathrm{MeV}$, and a high-intensity ring cyclotron with beam extraction at energies between 500 and $950 \mathrm{MeV}$.

\section{ACKNOWLEDGMENTS}

We thank Nada Fakhoury for her help in writing the MATHEMATICA ${ }^{\circledR}$ notebooks used for this work. Software has been written in " $\mathrm{C}$ " and been compiled with the GNU(C-C++ compiler on Scientific Linux. The figures have been generated with the cern library (PAW) and XFIG.

\section{APPENDIX: AN ALGEBRAIC METHOD FOR THE ANALYSIS OF ACCELERATOR LAYOUTS}

The layout of cyclotrons is just a special case of the general problem of the calculation of layouts, which is a special case of the geometry of curves in the plane. In the general case, a planar smooth curve can be described by a "state vector" $\psi$ that contains the coordinates and the direction derivatives $\boldsymbol{\psi}=\left(x, y, x^{\prime}, y^{\prime}\right)$, where $x$ and $y$ are the Cartesian coordinates of the orbit (planar curve) and $x^{\prime}=\frac{d x}{d s}$ and $y^{\prime}=\frac{d y}{d s}$ are the derivatives with respect to the path length $s$. By definition one has

$$
x^{\prime 2}+y^{\prime 2}=1,
$$

so that one may also write $\left(x^{\prime}, y^{\prime}\right)=(\cos \phi$, $\sin \phi)$ with the direction angle $\phi$ of the orbit.

In the hard-edge approximation, we assume that $\frac{1}{\rho}=\frac{q B}{p}$ is piecewise constant. In this case, a transfer matrix [32] method can be used and the solution is given by a transfer (or transport) matrix $\mathbf{M}(s)$ :

$$
\boldsymbol{\psi}(s)=\mathbf{M}(s) \boldsymbol{\psi}(0)
$$

where the matrix $\mathbf{M}$ is the product of the transfer matrices for the individual segments:

$$
\mathbf{M}(s)=\prod_{k=0}^{n-1} \mathbf{M}_{k} .
$$

In hard-edge approximation, there are basically two transfer matrices, the matrix $\mathbf{M}_{d}(L)$ for a drift of length $L$ and the matrix $\mathbf{M}_{b}(\rho, \alpha)$ for a bending magnet for a bending radius $\rho$ and angle $\alpha$ :

$$
\begin{gathered}
\mathbf{M}_{d}(L)=\exp (\mathbf{F} L)=\left(\begin{array}{cccc}
1 & 0 & L & 0 \\
0 & 1 & 0 & L \\
0 & 0 & 1 & 0 \\
0 & 0 & 0 & 1
\end{array}\right), \\
\mathbf{M}_{b}(\rho, \alpha)=\left(\begin{array}{cccc}
1 & 0 & \rho s & -\rho(1-c) \\
0 & 1 & \rho(1-c) & \rho s \\
0 & 0 & c & -s \\
0 & 0 & s & c
\end{array}\right),
\end{gathered}
$$

where $s=\sin \alpha, c=\cos \alpha$, and $\alpha=\frac{L}{\rho}$. A reverse bend (i.e. a bend into the opposite direction), is described by a negative radius and a negative angle, yielding a positive length $L=\alpha \rho$. If $\frac{1}{\rho}=0$, then the transfer matrix simplifies to the transfer matrix of a drift. These two matrices are sufficient to compute the layout of most accelerator beam lines. But they are also useful for the geometrical analysis of separate-sector cyclotrons with homogeneous field magnets in hard-edge approximation as described above.

In addition to the above transfer matrices, we will use the familiar coordinate rotation matrix $\mathbf{M}_{\text {rot }}(\theta)$ :

$$
\mathbf{M}_{\mathrm{rot}}(\theta)=\left(\begin{array}{cccc}
\cos \theta & -\sin \theta & 0 & 0 \\
\sin \theta & \cos \theta & 0 & 0 \\
0 & 0 & \cos \theta & -\sin \theta \\
0 & 0 & \sin \theta & \cos \theta
\end{array}\right) .
$$

If we consider an accelerator with $N$ equal sectors, thengiven an arbitrary starting position $\boldsymbol{\psi}(0)$ - the position and direction change after one sector relative to some center point $\boldsymbol{\psi}_{c}$ can be described by a rotation with an angle of $\theta=\frac{2 \pi}{N}$. Hence we may write

$$
\left[\boldsymbol{\psi}(L)-\boldsymbol{\psi}_{c}\right]=\mathbf{M}_{\mathrm{rot}}(\theta)\left[\boldsymbol{\psi}(0)-\boldsymbol{\psi}_{c}\right],
$$

so that by the use of Eq. (A2) one finds

$$
\begin{aligned}
\mathbf{M}_{\mathrm{sec}} \boldsymbol{\psi}(0)-\boldsymbol{\psi}_{c} & =\mathbf{M}_{\mathrm{rot}}(\theta)\left[\boldsymbol{\psi}(0)-\boldsymbol{\psi}_{c}\right], \\
{\left[\mathbf{M}_{\mathrm{sec}}-\mathbf{M}_{\mathrm{rot}}(\theta)\right] \boldsymbol{\psi}(0) } & =\left[\mathbf{1}-\mathbf{M}_{\mathrm{rot}}(\theta)\right] \boldsymbol{\psi}_{c} .
\end{aligned}
$$

The coordinates of the accelerator center can be obtained by

$$
\boldsymbol{\psi}_{c}=\left[\mathbf{1}-\mathbf{M}_{\mathrm{rot}}(\theta)\right]^{-1}\left[\mathbf{M}_{\mathrm{sec}}-\mathbf{M}_{\mathrm{rot}}(\theta)\right] \boldsymbol{\psi}(0) .
$$

The matrix $\mathbf{M}_{x}(\theta) \equiv\left[\mathbf{1}-\mathbf{M}_{\mathrm{rot}}(\theta)\right]^{-1}$ can be directly computed and is explicitly given by

$$
\begin{aligned}
\mathbf{M}_{x}(\theta) & =\frac{1}{2 \sin (\theta / 2)} \mathbf{M}_{\mathrm{rot}}(\pi / 2-\theta / 2) \\
& =\frac{1}{2}\left(\begin{array}{cccc}
1 & -\cot \theta / 2 & 0 & 0 \\
\cot \theta / 2 & 1 & 0 & 0 \\
0 & 0 & 1 & -\cot \theta / 2 \\
0 & 0 & \cot \theta / 2 & 1
\end{array}\right) .
\end{aligned}
$$

The computation of the center coordinates is therefore straightforward-and yields a result even if the matrix $\mathbf{M}_{\text {sec }}$ does not describe a "valid" sector. Such a nonvalid situation is found, if the "velocity" components of $\boldsymbol{\psi}_{c}$ do not vanish, which happens if the sum of the bending angles entering $\mathbf{M}_{\text {sec }}$ does not equal $\theta$.

In the following we use Eq. (A8) to compute the starting conditions for a cyclotron centered at $x_{c}=y_{c}=0$, i.e., the radius and direction of an equilibrium orbit. If we let the orbit start at $(0,0)$ in arbitrary direction, i.e., we choose for 
instance $\boldsymbol{\psi}(0)=(0,0,0,1)$, then the orbit with starting position $\boldsymbol{\psi}(0)-\boldsymbol{\psi}_{c}$ is centered. Hence, the starting position

$$
\boldsymbol{\psi}(0)=\left\{\mathbf{1}-\mathbf{M}_{x}(\theta)\left[\mathbf{M}_{\mathrm{sec}}-\mathbf{M}_{\mathrm{rot}}(\theta)\right]\right\}(0,0,0,1)^{T}
$$

is centered. The orbit still starts at an "arbitrary" angle $\theta_{0}$, i.e. $\boldsymbol{\psi}(0)$ as given by Eq. (A10) can be written as

$$
\boldsymbol{\psi}(0)=\left(R \cos \theta_{0}, R \sin \theta_{0}, 0,1\right)^{T}=\left(x_{0}, y_{0}, 0,1\right)^{T} .
$$

If one aims for a specific orientation of the orbit with respect to the coordinate system-for instance on the $x$ axis - then one may use the rotation matrix with $\theta_{0}=$ $\arctan \left(\frac{y_{0}}{x_{0}}\right)$ :

$$
\boldsymbol{\psi}_{0}=\mathbf{M}_{\mathrm{rot}}\left(-\theta_{0}\right) \boldsymbol{\psi}(0)=\left(R, 0, \frac{y_{0}}{R}, \frac{x_{0}}{R}\right)^{T} .
$$

The angular width of the magnet can then be calculated by computing the position angle of $\mathbf{M}_{b} \boldsymbol{\psi}(0)$.

If this method is applied to a separate-sector cyclotron with $N$ cells composed of a dipole with bending radius $r$ and bend angle $\frac{2 \pi}{N}$ and a drift of length $l$ as shown in Fig. 9, then one obtains from Eq. (A10)

$$
\boldsymbol{\psi}(0)=\left[r+l / 2 \cot \left(\frac{\pi}{N}\right), l / 2,0,1\right]^{T},
$$

so that the radius $R$ is given by

$$
R=\sqrt{\frac{l^{2}}{4}+\left(r+\frac{l}{2} \cot \frac{\pi}{N}\right)^{2}} .
$$

Using Eq. (A12), we sequentially obtain the coordinates $\boldsymbol{\psi}_{k}$ indicated in Fig. 9:

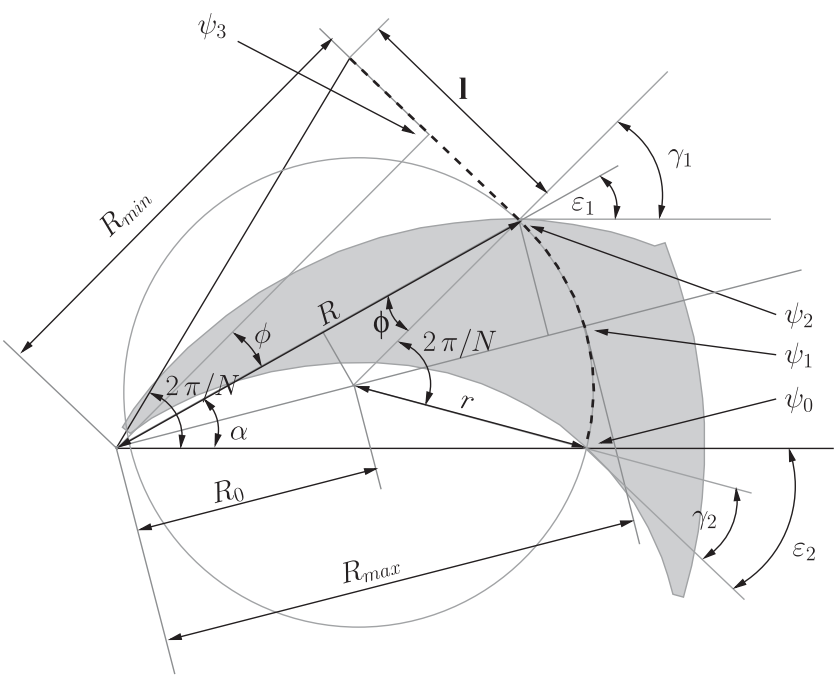

FIG. 9. Geometry of the cyclotron sector in case of a sector magnet (gray area) with a constant field along the closed orbit (shown as a thick dashed line). $\varepsilon_{1}$ and $\varepsilon_{2}$ are the spiral angles of the entrance and exit of the magnet. $\gamma_{1}$ and $\gamma_{2}$ are the angles between the sector entrance and exit and the orbit normal vector.
$\boldsymbol{\psi}_{0}=\mathbf{M}_{\mathrm{rot}}\left(-\theta_{0}\right) \boldsymbol{\psi}(0), \quad \boldsymbol{\psi}_{1}=\mathbf{M}_{b}(r, r \pi / N) \boldsymbol{\psi}_{0}$,

$\boldsymbol{\psi}_{2}=\mathbf{M}_{b}(r, r \pi / N) \boldsymbol{\psi}_{1}, \quad \boldsymbol{\psi}_{3}=\mathbf{M}_{d}(l / 2) \boldsymbol{\psi}_{2}$.

If $\psi_{k}^{i}$ is the $i$ th component of the state vector $\psi_{k}$, then

$R_{\max }=\sqrt{\left(\psi_{1}^{1}\right)^{2}+\left(\psi_{1}^{2}\right)^{2}}=r+\frac{l}{2 \sin (\pi / N)}$,

$R_{\min }=\sqrt{\left(\psi_{3}^{1}\right)^{2}+\left(\psi_{3}^{2}\right)^{2}}=r+\frac{l}{2} \cot (\pi / N)$.

The angle $\phi$ can be computed by

$$
\cot \phi=\frac{R_{\min }}{l / 2}=\frac{2 r}{l}+\cot \frac{\pi}{N} .
$$

Effectively, the angle $\phi$ equals the angle between $\psi(0)$ and $\psi_{0}$, so that one could derive Eq. (A17) directly from Eq. (A13).

The coordinates could also be derived from Fig. 9. The advantage of the algebraic method is that it gives an algorithm at hand that allows for the computation of essential geometric conditions directly from the parameters $r, N$, and $L$, without the need to analyze a "hand-made" drawing. Instead one might make use of the algebraic algorithm to generate the drawing.

[1] M. E. Rickey and R. Smythe, Nucl. Instrum. Methods 1819, 66 (1962).

[2] G. Gulbekyan, O.N. Borisov, and V.I. Kazacha, in Proceedings of HIAT2009 [http://accelconf.web.cern.ch/ AccelConf/HIAT2009/papers/d-02.pdf].

[3] Y. Jongen et al., Nucl. Instrum. Methods Phys. Res., Sect. A 624, 47 (2010).

[4] J. J. Yang, A. Adelmann, W. Barletta, L. Calabretta, A. Calanna, D. Campo, and J.M. Conrad, Nucl. Instrum. Methods Phys. Res., Sect. A 704, 84 (2013).

[5] L. Calabretta, G. Cuttone, M. Maggiore, M. Re, and D. Rifuggiato, Nucl. Instrum. Methods Phys. Res., Sect. A 562, 1009 (2006).

[6] O.N. Borisov, G.G. Gulbekyan, and D. Solivajs, in Proceedings of RuPAC XIX, Dubna 2004 [http://accelconf .web.cern.ch/accelconf/r04/papers/THBP02.PDF].

[7] D. Solivajs, O. N. Borisov, A. Gall, G. G. Gulbekian, J. Keniz, J. Kliman, S. Kralik, and M. Pavlovic, J. Electr. Eng. 55, 201 (2004).

[8] J.L. Ristic-Djurovic, Phys. Rev. ST Accel. Beams 4, 123501 (2001).

[9] E. Pedroni, D. Meer, C. Bula, S. Safai and S. Zenklusen, Eur. Phys. J. Plus 126, 66 (2011).

[10] J. W. Verba, W. Kündig, A. C. Paul, J. R. Richardson, and B. T. Wright, in Proceedings of the International Conference on Sector-Focused Cyclotrons and Meson Factories, CERN, Geneva, 1963, p. 101 [http://accelconf .web.cern.ch/AccelConf/c63/papers/cyc63c03.pdf].

[11] Y. Huang, A. Kumar, and S. Oh, in Proceedings of the 1987 Particle Accelerator Conference (IEEE, Washington, DC, 1987), p. 1881 [http://accelconf.web.cern.ch/ accelconf/p87/PDF/PAC1987_1881.PDF]. 
[12] M. K. Craddock and K. R. Symon, Reviews of Accelerator Science and Technology (World Scientific, Singapore, 2008), Vol. 1, p. 65.

[13] K. R. Symon, D. W. Kerst, L. W. Jones, L. J. Laslett, and K. M. Terwilliger, Phys. Rev. 103, 1837 (1956).

[14] J.I. M. Botman, M. K. Craddock, and C. J. Kost, in Proceedings of the 10th International Conference on Cyclotrons and their Applications, edited by F. Marti (IEEE, New York, 1984), pp. 32-35.

[15] M. K. Craddock and Y. N. Rao, in Proceedings of the 23rd Particle Accelerator Conference, Vancouver, Canada, 2009 (IEEE, Piscataway, NJ, 2009), p. 5044 [http://accelconf .web.cern.ch/AccelConf/PAC2009/papers/fr5rep114.pdf].

[16] H. A. Grunder and F. B. Selph, in Proceedings of the International Conference on Sector-Focused Cyclotrons and Meson Factories, CERN, Geneva, 1963, pp. 8-10 [http://accelconf.web.cern.ch/AccelConf/c63/papers/ cyc63a02.pdf].

[17] S. Antoine et al., Nucl. Instrum. Methods Phys. Res., Sect. A 602, 293 (2009).

[18] G. Schatz, Nucl. Instrum. Methods 72, 29 (1969).

[19] M. M. Gordon, Part. Accel. 16, 39 (1984) [http:// cds.cern.ch/record/1053175/files/p39.pdf].

[20] T. Zhang, J. Zhong, J. Wang, G. Dutto, G. Mackenzie, L. Root, X. Jia, Y. Bi, F. Guan, and S. Wei, in Proceedings of the 23rd Particle Accelerator Conference, Vancouver, Canada, 2009 (IEEE, Piscataway, NJ, 2009), p. 5035 [http://accelconf.web.cern.ch/AccelConf/ PAC2009/papers/fr5rep111.pdf \}.

[21] H.-U. Klein, C. Baumgarten, A. Geisler, J. Heese, A. Hobl, D. Krischel, M. Schillo, S. Schmidt, and
J. Timmer, Nucl. Instrum. Methods Phys. Res., Sect. B 241, 721 (2005).

[22] Y. Jongen, in Proceedings of CYCLOTRONS 2010, Lanzhou, China, pp. 398-403 [http://accelconf.web.cern .ch/AccelConf/Cyclotrons2010/papers/frm1cio01.pdf].

[23] B. Gottschalk, http://arxiv.org/abs/1204.4470v2.

[24] B. Gottschalk, Med. Phys. 37, 352 (2010).

[25] M. J. van Goethem, R van der Meer, H. W. Reist, and J. M. Schippers, Phys. Med. Biol. 54, 5831 (2009).

[26] J. H. Timmer, H. Röcken, T. Stephani, C. Baumgarten, and A. Geisler, Nucl. Instrum. Methods Phys. Res., Sect. A 568, 532 (2006).

[27] C. Rubbia et al., Report No. CERN-Report CERN/AT/ 95-44 [http://ab-atb-eet.web.cern.ch/ab-atb-eet/Papers/ EA/PDF/95-44.pdf].

[28] J. R. Alonso, High Current $\mathrm{H}_{2}^{+}$Cyclotrons for Neutrino Physics: The IsoDAR and DAESALUS Projects [http:// arxiv.org/abs/1210.3679].

[29] A. Adelmann et al., Cost-effective Design Options for IsoDAR [http://arxiv.org/abs/1210.4454].

[30] M. Seidel et al., in Proceedings of the International Particle Accelerator Conference, Kyoto, Japan (ICR, Kyoto, 2010), p. 1309 [http://accelconf.web.cern.ch/ AccelConf/IPAC10/papers/tuyra03.pdf].

[31] M.M. Gordon, in Proceedings of the International Conference on Sector-Focused Cyclotrons, Gatlinburg, TN, USA 1966 [IEEE Trans. Nucl. Sci. 13, 48 (1966)] [http:// accelconf.web.cern.ch/AccelConf/c66/papers/b-001.pdf].

[32] The transfer matrices used to describe layouts should not be confused with the well-known transfer matrices that are used to describe the beam optics. 